\title{
The optimal asset allocation of the main types of pension funds: a unified framework
}

\author{
Katarzyna Romaniuk
}

Received: 23 September 2006/Revised: 16 May 2007/Published online: 17 August 2007

(C) The Geneva Association 2007

\begin{abstract}
The existing literature deals with the optimal investment strategy of defined benefit (DB) or defined contribution (DC) pension plans. This article's objective is to compare the optimal policies of different types of pension plans. This is done by first defining an original framework, which is based on the distinction between the nature of the guarantee-which can be internal or external-offered by or to a pension fund. This framework allows to establish links between optimization programs of DC, DB and targeted money purchase schemes. The case of an internal guarantee appears as a standard portfolio insurer's problem. The second kind of guarantee, not analyzed in the literature yet with regard to the resulting optimal policy, is characterized by the existence of an option in the final wealth definition. Four funds are present in the internal guarantee optimal allocation: the speculative component, the preference independent guarantee- and contribution-hedge terms and the preference dependent state variable-hedge fund. The external guarantee program, solved with an original method using the principles of standard options theory, yields an optimal policy incorporating the delta of the option embodied in the final wealth definition. The conclusion is that the resulting optimal portfolio policy becomes riskier.
\end{abstract}

Keywords Pension funds · Defined benefit - Defined contribution · Asset allocation · Internal and external guarantees · Stochastic dynamic programming · Options theory

JEL Classification $\mathrm{G} 23 \cdot \mathrm{G} 11 \cdot \mathrm{C} 61$

K. Romaniuk $(\bowtie)$

PRISM, University of Paris 1 Panthéon-Sorbonne, UFR 06, 1, rue Victor Cousin, Paris 75005,

France

e-mail: romaniuk@univ-paris1.fr 


\section{Introduction}

Pension funds cannot generally be managed in the way standard funds are. The heavy regulation governing the pension fund functioning is one of the reasons. Defined benefit (DB) funds often have to observe some rules as to the funding of future liabilities. As the pension fund performance largely determines the standard of living in the retirement period, the defined contribution (DC) fund management can be subject to minimum performance constraints.

This article seeks to determine the optimal asset allocation for the main types of pension plans, in a continuous-time stochastic framework. We consider the case of a fund manager maximizing the expected utility of final wealth, his control variable being defined as the proportion of wealth to invest in the risky asset.

This framework, based on the seminal papers by Merton [1971] — for the stochastic dynamic programming approach—and Cox and Huang [1989, 1991]—for the martingale approach, is often used in the literature dealing with the pension fund asset allocation issue. The most obvious classification of the papers results from the type of scheme analyzed: The articles study DC or DB plans. In a DB scheme, the participant receives a pension depending mainly on his wage (final or averaged) and the number of years of service. In a DC scheme, his pension depends solely on the value of the assets accumulated at the time of retirement. As outlined by Blake [1998] and Boulier et al. [2001], the DC fund program strongly resembles an individual saving decision, whereas the DB fund management can be characterized as an asset-liability management issue.

In a DB scheme, the fund manager is obliged to conduct a policy guaranteeing that the final asset value lies above the final liability value, which leads the investor to maximize the expected utility of the surplus of assets over the liabilities of the fund. A new term then appears in the optimization program solution: a preference independent liability-hedge fund [Sundaresan and Zapatero, 1997; Rudolf and Ziemba, 2004].

Concerning the DC fund management issue, the general model of Menoncin [2002] constitutes the starting point of the first branch of the literature. The main contribution of the paper is the analysis of the role of background variables, which are outside the financial market, yet affect directly the individual's wealth. Their existence leads to the presence, in the optimal allocation, of a preference independent term, linked to the background variable diffusion parameter. Battocchio and Menoncin [2004] focus on two background risks: inflation and salary. While these authors analyze the accumulation phase, Menoncin and Scaillet [2003] and Battocchio et al. [2007] generalize this approach by treating both the accumulation and decumulation phases in a unique setting. Although it is not based on the model of Menoncin [2002], the framework by Cairns et al. [2006] resembles the one adopted in the previous papers, except for the use of a salaryrelated numeraire as argument of the utility function: Such a definition models the fact that the participant would like to preserve his standard of living after retirement. The second branch of the literature considers the problem of a fund subject to a minimum guarantee to be met at retirement [Boulier et al., 2001; Deelstra et al., 2003]. 
Menoncin and Scaillet [2003] stress that their model (which appears as the most general and complete) can be applied to both DB and DC plans: As contributions and pensions are set as stochastic variables, by equating the diffusion term of pensions (respectively contributions) to zero, the framework becomes the one of a DB (respectively DC) plan. Yet this distinction is questionable when the salary is assumed to be stochastic: Neither the DB pension, which depends in general on the level of the final salary, nor the DC contributions, which generally take the form of a proportion of salary (in either a DB or a DC plan), can be then considered as deterministic.

One can thus conclude that there exists a need for developing a unified framework in which the optimal asset allocation policies of the main types of pension funds could be compared.

This is done by first proposing a new classification based on the nature of the guarantee-internal or external-linked to the pension fund performance. In the internal guarantee case, the fund itself is obliged to generate a minimum return. When an external guarantee is granted, an institution external to the fund covers the possible positive difference between the guaranteed return and the one actually generated. The nature of the guarantee then determines the form of the optimization program: Whereas the program characteristic of an internal guarantee resembles the one of a portfolio insurer, the one linked to an external guarantee takes an original form, characterized by the presence of an option in the final wealth definition. The possible programs of DC, DB and targeted money purchase (TMP) schemes are then discussed, with regard to the person responsible for the deficit, the nature of the guarantee and the presence of a surplus sharing rule, and the links between the optimization programs of the different types of plans are clearly identified.

Finally, the optimization programs are solved with the stochastic dynamic programming technique. The internal guarantee optimal strategy is characterized by four components : the speculative term, two preference independent terms- the guarantee-hedge fund and the wage-hedge fund (resulting from the contribution process) and a preference dependent state variable-hedge component. The solution of the external guarantee case, obtained with an original method based on the principles of standard options theory, shows that the investor adopts a riskier policy: As the speculative term is divided by the delta of the call incorporated in the final wealth definition, its absolute value increases when compared with the internal guarantee case.

The article is organized as follows. Section 2 defines the optimization programs of the main types of pension funds. The model is presented in Section 3. Optimal policies are derived and discussed in Section 4. Section 5 concludes.

\section{The optimization programs of the main types of pension funds}

The chosen setting builds on the distinction between internal and external guarantees. Internal guarantees concern a minimum performance constraint: The fund has to generate a minimum return. External guarantees are given by an 
institution independent from the fund: When the guaranteed return exceeds the one actually generated, the institution covers the difference.

One analyzes the optimization programs of three kinds of pension plans: the DC plan, which offers a pension corresponding to the fund asset value $A$ at the time of retirement $T$, the DB plan, whose pension equals the retirement value of the fund liabilities $L$, and the TMP scheme, where the participant receives a pension equal to the maximum of the DC pension and the targeted one. Three subcases are considered in the case of a DC fund: without guarantee and with an internal or external guarantee, both guarantees being denoted by $G$. In the case of a DB fund, the article studies the subcases without guarantee or with a (possibly partial) guarantee, the guarantee $G$ possibly offered in a DB fund being in fact equal to the fund liabilities $L$. For the two DB subcases, one analyzes the consequences of a rule of surplus sharing between the employee and the employer: The participant receives $\alpha$ and the firm $1-\alpha$ of the generated surplus, with $\alpha$ a constant satisfying $0<\alpha<1$.

It is throughout assumed that the participant (or the employee) and the manager are a single agent, the purpose of the manager being defined as the determining of the optimal strategy from the participant's point of view. The employer (or the firm) does not intervene in the definition of the investment strategy.

The optimization programs incorporate three constraints. First, the argument of the utility function must be strictly positive at the initial date. One takes into account the future flow of contributions by adding, to the initial value of the fund capital, the expected discounted value of future contributions [Merton, 1990]. Furthermore, the argument of the utility function must be non-negative at the time of retirement. Finally, the dynamics of the variables form the last constraint.

Depending on the case under scrutiny, the optimization program of the fund manager, on a date $t$ preceding the retirement date $T$, can take two forms:

$$
\operatorname{Max} E_{t}[U(A(T)-G(T))]
$$

or

$$
\operatorname{Max} E_{t}[U(G(T)+\alpha \operatorname{Max}(A(T)-G(T), 0)]
$$

with $E_{t}$ the expectation, conditional on the information available in $t, U$ the utility function and $0<\alpha \leq 1$.

Let us emphasize that the presence of a surplus sharing rule is characterized by $0<\alpha<1$, while when $\alpha=1$, the programs just defined are characteristic of the general cases of an internal and an external guarantee respectively. The manager's choice between these two types of programs results from the nature of the person directly responsible for the possible deficit (which occurs when $A(T)<G(T)$ ). If the participant, the fund or an institution influencing management decisions is responsible, program (1) is chosen. Program (2) characterizes a guarantee given by an institution external to the fund and its management decisions.

Program (1) applies for numerous configurations.

First, the case $G(T)=0$ corresponds to the one of a DC fund without guarantee. The program then takes the standard form, proposed by Merton [1971]: $\operatorname{Max} E_{t}[U(A(T))]$. 
Second, a DC fund with an internal guarantee uses this program. The fund asset value must be at least $G(T)$ at the date of retirement. The program becomes $\operatorname{Max} E_{t}[U(A(T))]$ under the solvency constraint $A(T) \geq G(T)$. Following Basak [2002], we adopt the program (1) formulation. ${ }^{1}$

Third, when $G(T)=L(T)$, the program is characteristic of a DB fund without guarantee or with a partial guarantee, and in the same time using a surplus sharing rule. When no guarantee is given, the participant bears the consequences of a possible deficit, while the possible surplus is owned by the firm. The participant will thus receive the minimum of $L(T)$ and $A(T)$, so that he is not preoccupied about the generation of a surplus. He will thus invest his entire wealth (the initial fund capital, as well as the contributions which accumulate into the fund) in the asset $L$, directly or by replication. ${ }^{2}$ Yet when a surplus sharing rule is introduced, the participant's program will be $\operatorname{Max} E_{t}[U(L(T)+\alpha \operatorname{Max}(A(T)-L(T), 0))]$ under the fund solvency constraint $A(T) \geq L(T)$, with $0<\alpha<1$. The solvency constraint is then determining for the form of the participant's program. His main purpose is the generation of a surplus: The participant will then be certain to receive at least the liability value at the date of retirement, and will in the same time benefit from a proportion of the generated surplus. Program (1), with $G(T)=L(T)$, thus applies. In the case of a partial guarantee, the employee prefers to obtain $L(T)$ rather than a proportion of $L(T)$, so that the optimization program includes the solvency constraint. The case of a DB fund without guarantee applies: If the participant does not benefit from the surplus, he will invest in $L$; In the case of a surplus sharing rule, he will maximize $E_{t}[U(A(T)-L(T)]$.

Fourth, the allocation policy of a DB fund with a guarantee, and without a surplus sharing rule, is defined by this program. The employer now owns the possible surplus, but is in the same time responsible for the payment of the possible deficit. As the participant knows that he will receive the liability value at the retirement date, independently of the fund asset value, this situation could become dangerous for the employer, because the participant is not preoccupied about the adopted strategy. To neutralize the risk of an inappropriate allocation, the firm will take the responsibility of defining the fund policy. The employer's program writes: $\operatorname{Max} E_{t}[U(A(T)-L(T))]$.

Finally, Max $E_{t}[U(A(T)-G(T))]$ defines the program of a TMP fund targeting the value $G(T)$ at the date of retirement. The TMP plan participant maximizes

\footnotetext{
${ }^{1}$ In the program (1) formulation, the utility function argument implicitly contains the condition of a final wealth above the chosen level, provided that $\lim _{A \rightarrow G^{+}} U^{\prime}(A-G)=+\infty$ [Karatzas et al., 1986; Merton, 1990], a condition which we impose on the utility function. According to Basak [2002], this alternative modeling approach has two advantages, when compared with the initial program formulation. Beyond the fact that the calculations are largely facilitated because the explicit constraint disappears, the properties of the utility function describe the portfolio insurer's preferences in a more exact way. Basak [2002] stresses that, in the formulation using the explicit constraint, when the terminal wealth of the insurer hits the guaranteed level, his marginal indirect utility of wealth jumps discontinuously to infinity. In the proposed formulation (and under the condition on the utility function given above), the marginal utility goes smoothly to infinity at the floor, so that the optimal final wealth is automatically above the final guarantee value.

2 The feasibility of this policy results from the completeness of the financial market and from the initial constraint, which states that the initial fund capital and the expected discounted value of future contributions strictly exceed the initial value of the liabilities.
} 
$E_{t}[U(\operatorname{Max}(A(T), G(T)]$ under the solvency constraint $A(T) \geq G(T)$, because the policy must be conducted in a way guaranteeing that he receives at least $G(T)$ at the retirement date. Because of this solvency constraint, the participant will always receive $A(T)$. One more time, the solvency constraint is determining for the form of the participant's program. His main purpose is the generation of a surplus, which will guarantee that the constraint is met, and in the same time an amount received $A(T)$ larger than the minimum $G(T)$. The program thus takes the form (1).

As to program (2), when $\alpha=1$, it corresponds to a DC fund with an external guarantee. The participant's program then is $\operatorname{Max} E_{t}[U(\operatorname{Max}(A(T), G(T))]$ without the solvency constraint, because the participant knows that he will receive the maximum of $A(T)$ and $G(T)$, whatever the relative value of $A(T)$ with respect to $G(T)$.

When $0<\alpha<1$ and $G(T)=L(T)$, the program is the one of a DB fund characterized by the presence of a guarantee and a surplus sharing rule.

\section{The model}

\subsection{Assumptions}

The pension fund manager can invest in $N$ risky assets. The dynamics of the price $S_{i}(t)$ of the $i$ th risky asset is described by the following stochastic differential equation (SDE):

$$
\mathrm{d} S_{i}(t)=S_{i}(t) \mu_{S_{i}}(t, Z(t)) \mathrm{d} t+S_{i}(t) \sigma_{S_{i}}(t, Z(t)) \mathrm{d} B(t)
$$

where $\mu_{S_{i}}(t, Z(t))$ denotes a bounded function of $t$ and $Z, \sigma_{S_{i}}(t, Z(t))$ a bounded $(1 \times M)$ vector valued function of $t$ and $Z, B(t)$ an $(M \times 1)$-dimensional Wiener process in $R^{M}, Z(t)$ a $(K \times 1)$-dimensional vector of state variables.

The compacted form of the dynamics of the $N$ risky asset prices then writes:

$$
\mathrm{d} S(t)=I_{S} \mu_{S}(t, Z(t)) \mathrm{d} t+I_{S} \sigma_{S}(t, Z(t)) \mathrm{d} B(t)
$$

with $I_{S}$ defined as an $(N \times N)$ diagonal matrix valued function of $S(t)$ with $S_{i}(t)$ as its $i$ th diagonal element, $\mu_{S}(t, Z(t))$ an $(N \times 1)$-dimensional vector with $\mu_{S_{i}}(t, Z(t))$ as its $i$ th element, $\sigma_{S}(t, Z(t))$ an $(N \times M)$ matrix valued function with $\sigma_{S_{i}}(t, Z(t))$ as its $i$ th element.

$K$ stochastic state variables influence the evolution of the economy. Their dynamics obey:

$$
\mathrm{d} Z(t)=I_{Z} \mu_{Z}(t, Z(t)) \mathrm{d} t+I_{Z} \sigma_{Z}(t, Z(t)) \mathrm{d} B(t)
$$

where $I_{Z}$ stands for a $(K \times K)$ diagonal matrix valued function of $Z(t), \mu_{Z}(t, Z(t))$ a bounded $(K \times 1)$ vector valued function of $t$ and $Z, \sigma_{Z}(t, Z(t))$ a bounded $(K \times M)$ matrix valued function of $t$ and $Z^{3}$

\footnotetext{
${ }^{3}$ The definition of the dynamics of the risky assets and the state variables is comparable to the framework proposed by Lioui and Poncet [2003].
} 
The pension fund manager can also invest in the riskless asset, whose price $\eta(t)$ evolves according to the dynamics:

$$
\mathrm{d} \eta(t)=\eta(t) r(t, Z(t)) \mathrm{d} t
$$

where $r(t, Z(t))$ is defined as the instantaneously riskless interest rate, which is assumed to depend on the state variables $Z{ }^{4}$

The pension fund management can be subject to a minimum guarantee which is to be met internally by the fund or is offered by an external institution.

A contribution is brought continuously into the fund. It corresponds to a constant proportion $\zeta$ of the employee's salary.

The dynamics of the guarantee $G$ and the wage $Y$ take the form of the following SDEs:

$$
\begin{aligned}
\mathrm{d} G(t) & =G(t) \mu_{G}(t, Z(t)) \mathrm{d} t+G(t) \sigma_{G}(t, Z(t)) \mathrm{d} B(t) \\
\mathrm{d} Y(t) & =Y(t) \mu_{Y}(t, Z(t)) \mathrm{d} t+Y(t) \sigma_{Y}(t, Z(t)) \mathrm{d} B(t)
\end{aligned}
$$

where $\mu_{G}(t, Z(t))$ and $\mu_{Y}(t, Z(t))$ denote bounded functions of $t$ and $Z, \sigma_{G}(t, Z(t))$ and $\sigma_{Y}(t, Z(t))$ bounded $(1 \times M)$ vector valued functions of $t$ and $Z$.

Standard assumptions about the financial market apply. In particular, it is assumed to be complete, which is mathematically translated by $N=M$.

\subsection{The investor's program}

The utility function of the participant, assumed increasing and concave in $X$, takes the terminal form $U(X(T))$, where $X(T)=A(T)-G(T)$ or $X(T)=G(T)+\alpha \operatorname{Max}(-$ $A(T)-G(T), 0)$ with $0<\alpha \leq 1$, depending on the case under scrutiny. It is further assumed that the utility function respects the Inada conditions: $\lim _{X \rightarrow \infty} U_{X}=0$ and $\lim _{X \rightarrow 0} U_{X}=\infty$, with $U_{X}$ the derivative of $U$ with respect to $X$.

The manager's program writes:

$$
\begin{aligned}
& \operatorname{Max}_{x_{S}} E_{t}[U(X(T))] \\
& \text { s.c. } \mathrm{d} X(t)=X(t) \mu_{X}\left(t, x_{S}, X(t), Z(t)\right) \mathrm{d} t+X(t) \sigma_{X}\left(t, x_{S}, X(t), Z(t)\right) \mathrm{d} B(t) \\
& \mathrm{d} Z(t)=I_{Z} \mu_{Z}(t, Z(t)) \mathrm{d} t+I_{Z} \sigma_{Z}(t, Z(t)) \mathrm{d} B(t) \\
& X(0)>0 \\
& X(T) \geq 0
\end{aligned}
$$

with $x_{S}$ an $(N \times 1)$ vector of the proportions of $X$ invested in the risky assets $\left(x_{S_{i}}\right.$ being the proportion of $X$ invested in the $i$ th risky asset), the variable $X$ dynamics being written in a general form, where $\mu_{X}\left(t, x_{S}, X(t), Z(t)\right)$ stands for a function of $t$,

\footnotetext{
${ }^{4}$ Menoncin [2002] opts for a similar modeling of the riskless asset dynamics.
} 
$x_{S}, X$ and $Z$ and $\sigma_{X}\left(t, x_{S}, X(t), Z(t)\right)$ a $(1 \times M)$ vector valued function of $t, x_{S}, X$ and $Z^{5}$

\section{Definition of the optimal policy}

Stochastic dynamic programming is used when solving the optimization programs.

4.1 Solution in the general case

Let us define the indirect utility function $J$, increasing and strictly concave in $X$, by the following equation:

$$
J(t, X(t), Z(t)) \equiv \max _{x_{S}} E_{t}[U(X(T))]
$$

The Hamilton-Jacobi-Bellman (HJB) equation takes the form:

$$
\max _{x_{S}} D J=0
$$

where $D$ denotes the Dynkin operator, the Dynkin of $J$ being defined by:

$$
D J=J_{t}+J_{X} X \mu_{X}+\frac{1}{2} J_{X X} X^{2} \Sigma_{X X}+\left(I_{Z} \mu_{Z}\right)^{\prime} J_{Z}+\frac{1}{2} \operatorname{tr}\left(I_{Z} \Sigma_{Z Z} I_{Z}^{\prime} J_{Z Z}\right)+X \Sigma_{X Z} I_{Z}^{\prime} J_{X Z}
$$

where subscripts on $J$ denote partial derivatives ${ }^{6}$, the prime ' stands for a transpose and $\Sigma_{i j} \equiv \sigma_{i} \sigma_{j}^{\prime}$ the covariance matrix of the variables $i$ and $j$, the dependence with respect to time $t, x_{S}, X$ and $Z$ having been omitted for ease of exposition.

From now on, we shall omit this kind of dependence, except when a risk of confusion occurs.

4.2 The case of an internal guarantee

\subsubsection{Optimization program solution}

The fund assets $A$ are invested in the risky assets $S_{i}(i=1,2, \ldots, N)$ and in the riskless asset $\eta, X_{S_{i}}$ denoting the number of asset $S_{i}$ acquired and $X_{\eta}$ the amount of riskless asset held.

In the case of an internal guarantee, $X=A-G$. The composition of $X$ follows:

\footnotetext{
$5 \mu_{X}$ and $\sigma_{X}$, which have different formulations depending on the variable $X$ definition chosen, will be precisely defined later.

${ }^{6}$ We assume that the function $J$ is twice differentiable with respect to $X$ and $Z$. Let us note that, in the literature, authors often set a specific form for the function $J$, allowing to meet the assumptions and to derive explicit solutions [Menoncin, 2002].
} 


$$
X=\sum_{i=1}^{N} X_{S_{i}} S_{i}+X_{\eta} \eta-G
$$

The variable $X$ is invested in the risky asset $S_{i}$ in the proportion $x_{S_{i}} \equiv \frac{X_{S_{i}} S_{i}}{X}$, in the riskless asset $\eta$ in the proportion $x_{\eta} \equiv \frac{X_{\eta} \eta}{X}$ and (negatively) in the guarantee $G$ in the proportion $x_{G} \equiv \frac{G}{X}$, with the following identity: $\sum_{i=1} x_{S_{i}}+x_{\eta}-x_{G}=1$.
The variable $X$ dynamics writes:

$$
\frac{\mathrm{d} X}{X}=\sum_{i=1}^{N} x_{S_{i}} \frac{\mathrm{d} S_{i}}{S_{i}}+x_{\eta} \frac{\mathrm{d} \eta}{\eta}+\zeta \frac{Y}{X} \frac{\mathrm{d} Y}{Y}-x_{G} \frac{\mathrm{d} G}{G}
$$

the term $\zeta \frac{Y}{X} \frac{\mathrm{d} Y}{Y}$ resulting from the accumulation of the contribution process. ${ }^{7}$ These contributions take the form of a proportion $\zeta$ of the employee's salary. They accumulate continuously into the pension fund assets. As soon as the contributions are integrated, they are invested in the assets $S_{i}$ and $\eta$.

${ }_{N}$ By replacing the dynamics (3), (6), (7) and (8) and by using the identity $\sum_{i=1}^{N} x_{S_{i}}+x_{\eta}-x_{G}=1$, one obtains:

$$
\begin{aligned}
\frac{\mathrm{d} X}{X}= & {\left[x_{S}^{\prime}\left(\mu_{S}-r 1_{N}\right)+r+\zeta \frac{Y}{X} \mu_{Y}-x_{G}\left(\mu_{G}-r\right)\right] \mathrm{d} t } \\
& +\left[x_{S}^{\prime} \sigma_{S}+\zeta \frac{Y}{X} \sigma_{Y}-x_{G} \sigma_{G}\right] \mathrm{d} B
\end{aligned}
$$

The system of first order conditions, obtained by deriving the Dynkin of $J$ with respect to $x_{S}$, writes:

$$
0_{N}=\left(\mu_{S}-r 1_{N}\right) J_{X} X+\left(\Sigma_{S S} x_{S}+\Sigma_{S Y} \zeta \frac{Y}{X}-\Sigma_{S G} x_{G}\right) J_{X X} X^{2}+\Sigma_{S Z} I_{Z}^{\prime} J_{X Z} X
$$

The optimal vector of proportions $x_{S}$ follows:

$$
\begin{aligned}
x_{S}= & -\left(\Sigma_{S S}\right)^{-1}\left(\mu_{S}-r 1_{N}\right) \frac{J_{X}}{J_{X X} X} \\
& -\left(\Sigma_{S S}\right)^{-1} \Sigma_{S Y} \zeta \frac{Y}{X}+\left(\Sigma_{S S}\right)^{-1} \Sigma_{S G} x_{G}-\left(\Sigma_{S S}\right)^{-1} \Sigma_{S Z} I_{Z}^{\prime} J_{X Z} \frac{1}{J_{X X} X}
\end{aligned}
$$

\subsubsection{Result interpretation}

Four funds form together the optimal investment strategy: the usual speculative fund, two preference independent terms - the contribution- and the guarantee-hedge portfolios and a preference dependent state variable-hedge component.

The standard mean-variance speculative component goes back to Merton [1971]. Yet his framework is the one where $G=0$. As a consequence, instead of defining

\footnotetext{
7 Battocchio and Menoncin [2004] propose a similar modeling.
} 
the optimal proportion to invest in the risky assets with respect to $A-G$, Merton [1971] defines it relative to $A$. When one sets our speculative fund with respect to $A$ (i.e. as a proportion of $A$, and not of $X$ anymore), one obtains $-\left(\Sigma_{S S}\right)^{-1}\left(\mu_{S}-r 1_{N}\right) \frac{J_{X}}{J_{X X} A}$ : When going from the case without guarantee to the one with guarantee, the speculative fund is modified by exchanging $\frac{J_{A}}{J_{A A}}$ for $\frac{J_{X}}{J_{X X}}$. A utility function of the CRRA type thus gives divergent speculative fund formulations, depending on whether a guarantee is granted or not.

Preference independent, the second term hedges against variations in the wage. The third component constitutes a preference independent hedge against variations in the guarantee. By writing it with respect to $A$, one obtains $\left(\Sigma_{S S}\right)^{-1} \Sigma_{S G} \frac{G}{A}$. By further noting that, in the case of a DB fund, the guarantee equals the liabilities of the fund, which take the form of the salary multiplied by a factor $\gamma: G(T)=L(T)=$ $\gamma Y(T)$, one can rewrite the guarantee-hedge fund: $\left(\Sigma_{S S}\right)^{-1} \Sigma_{S Y} \frac{\gamma Y}{A}$. The liabilityhedge term thus depends on the ratio $\frac{L}{A}$, which is representative of the financial health of the fund [Rudolf and Ziemba, 2004]. The higher this ratio, and thus the worse the fund financial health, the higher the value of this hedge portfolio (in absolute value). Let us finally note that, in the case of a DB fund, the salary appears in both the contribution and the pension definition. As a consequence, the second and the third terms of the optimal strategy depend on the variable $Y$. One concludes that, in a DB fund, the role, for determining the optimal investment strategy, of the salary variable is more pronounced than in the standard case.

The fourth portfolio component defines a preference dependent state variablehedge fund, which is standard when introducing state variables influencing the main variable dynamics.

\subsection{The case of an external guarantee}

\subsubsection{Optimization program solution}

The case of an external guarantee has not been analyzed in the literature yet. We propose an original method for solving the optimization program, based on standard results of options theory.

The variable $X$ now takes the following form: $X(T)=G(T)+\alpha \operatorname{Max}(-$ $A(T)-G(T), 0)$, with $0<\alpha \leq 1$. Let us denote by $C$ the call incorporated in $X$, defined by its payoff at maturity $T: C(T)=\operatorname{Max}(A(T)-G(T), 0)$. $C$ defines an exchange option, whose valuation method in the benchmark framework of Black and Scholes [1973] has been proposed by Margrabe [1978]. The variable $X$ can be reformulated:

$$
X(T)=G(T)+\alpha C(T)
$$

The variable $X$ is invested in the guarantee $G$ and in the call $C$, in the proportions $x_{G}$ and $x_{C} \equiv \frac{C}{X}$ respectively, with $x_{G}+\alpha x_{C}=1$. The dynamics of $X$ then writes:

$$
\frac{\mathrm{d} X}{X}=x_{G} \frac{\mathrm{d} G}{G}+\alpha x_{C} \frac{\mathrm{d} C}{C}
$$


Let us first define the dynamics of the call $C\left(t, A, G, Z_{1}, Z_{2}, \ldots, Z_{K}\right)$. By applying Ito's lemma to the function $C$, one obtains:

$$
\mathrm{d} C=C_{t} \mathrm{~d} t+\sum_{j} C_{j} \mathrm{~d} j+\frac{1}{2} \sum_{j} \sum_{k} C_{j k} \mathrm{~d} j \mathrm{~d} k
$$

with $j, k=\left\{A, G, Z_{1}, Z_{2}, \ldots, Z_{K}\right\}$ and $C_{j}$ denoting the partial derivative of the call with respect to the variable $j, C_{j k}$ its second derivative with respect to $j$ and $k$.

By adapting the non-arbitrage portfolio of Black and Scholes [1973] to the case of an option depending on more than one stochastic variable, the risk-free portfolio $\pi$ takes the form:

$$
\pi=-C+\sum_{j} C_{j} j
$$

Due to the self-financing condition:

$$
\mathrm{d} \pi=-\mathrm{d} C+\sum_{j} C_{j} \mathrm{~d} j
$$

By combining the equations (16) and (18), one obtains:

$$
\mathrm{d} \pi=-C_{t} \mathrm{~d} t-\frac{1}{2} \sum_{j} \sum_{k} C_{j k} \mathrm{~d} j \mathrm{~d} k
$$

The portfolio $\pi$ being riskless, it earns the riskless rate $r$. Thus the dynamics of $\pi$ writes:

$$
\mathrm{d} \pi=r \pi \mathrm{d} t
$$

The combination of the equations (17), (19) and (20) implies:

$$
-C_{t} \mathrm{~d} t-\frac{1}{2} \sum_{j} \sum_{k} C_{j k} \mathrm{~d} j \mathrm{~d} k=r\left(-C+\sum_{j} C_{j j}\right) \mathrm{d} t
$$

By replacing in the equation (16), the dynamics of the call can be rewritten in the following form: ${ }^{8}$

\footnotetext{
${ }^{8}$ The referee proposed a more direct method for obtaining these call dynamics. Instead of creating a nonarbitrage portfolio of the kind proposed by Black and Scholes [1973], one just needs to note that, under the risk neutral probability measure, the drift of the call dynamics must be $r$. Then simply applying Girsanov's theorem, one obtains the proposed dynamics.
} 


$$
\frac{\mathrm{d} C}{C}=\left[r+\sum_{j} C_{j} \frac{j}{C}\left(\mu_{j}-r\right)\right] \mathrm{d} t+\sum_{j} C_{j} \frac{j}{C} \sigma_{j} \mathrm{~d} B
$$

Let us derive the dynamics of the assets $A$. The composition of $A$ writes: $A=\sum_{i=1}^{N} X_{S_{i}} S_{i}+X_{\eta} \eta . A$ is invested in the risky assets $S_{i}$ and $\eta$, in the proportions $\chi_{S_{i}} \equiv \frac{X_{S_{i}} S_{i}}{A}$ and $\chi_{\eta} \equiv \frac{X_{\eta} \eta}{A}$ respectively, with the following identity: $\sum_{i=1}^{N} \chi_{S_{i}}+\chi_{\eta}=1$. While $x_{S_{i}}, x_{\eta}$ and $x_{G}$ were defined as proportions of $X, \chi_{S_{i}}$ and $\chi_{\eta}$ are defined as proportions of $A, x_{S_{i}}$ and $\chi_{S_{i}}$ being related by $\chi_{S_{i}}=x_{S_{i}} \frac{X}{A}$. Thus the dynamics of $A$ writes:

$$
\frac{\mathrm{d} A}{A}=\sum_{i=1}^{N} \chi_{S_{i}} \frac{\mathrm{d} S_{i}}{S_{i}}+\chi_{\eta} \frac{\mathrm{d} \eta}{\eta}+\zeta \frac{Y}{A} \frac{\mathrm{d} Y}{Y}
$$

the introduction of the term $\zeta \frac{Y}{A} \frac{\mathrm{d} Y}{Y}$ resulting from the contribution accumulation. ${ }_{N}$ By replacing the dynamics (3), (6) and (8) and by using the identity $\sum_{i=1}^{N} \chi_{S_{i}}+\chi_{\eta}=1$, the dynamics of $A$ becomes:

$$
\frac{\mathrm{d} A}{A}=\left[\chi_{S}^{\prime}\left(\mu_{S}-r 1_{N}\right)+r+\zeta \frac{Y}{A} \mu_{Y}\right] \mathrm{d} t+\left[\chi_{S}^{\prime} \sigma_{S}+\zeta \frac{Y}{A} \sigma_{Y}\right] \mathrm{d} B
$$

By combining the equations (21) and (22), the dynamics of the call writes:

$$
\begin{aligned}
\frac{\mathrm{d} C}{C}= & {\left[r+C_{A} \frac{A}{C}\left[\chi_{S}^{\prime}\left(\mu_{S}-r 1_{N}\right)+\zeta \frac{Y}{A} \mu_{Y}\right]+C_{G} \frac{G}{C}\left(\mu_{G}-r\right)+\frac{1}{C} C_{Z}^{\prime} I_{Z}\left(\mu_{Z}-r 1_{K}\right)\right] \mathrm{d} t } \\
& +\left[C_{A} \frac{A}{C}\left[\chi_{S}^{\prime} \sigma_{S}+\zeta \frac{Y}{A} \sigma_{Y}\right]+C_{G} \frac{G}{C} \sigma_{G}+\frac{1}{C} C_{Z}^{\prime} I_{Z} \sigma_{Z}\right] \mathrm{d} B
\end{aligned}
$$

Let us remind that $\frac{\mathrm{d} X}{X}=x_{G} \frac{\mathrm{d} G}{G}+\alpha x_{C} \frac{\mathrm{d} C}{C}$. By using the definitions of $x_{G}$ and $x_{C}$, the identities $x_{G}+\alpha x_{C}=1$ and $\chi_{S_{i}}=x_{S_{i}} \frac{X}{A}$, and after rearranging terms, one finally obtains the dynamics of the variable $X$ :

$$
\begin{aligned}
\frac{\mathrm{d} X}{X}= & {\left[r+\alpha C_{A} x_{S}^{\prime}\left(\mu_{S}-r 1_{N}\right)+\alpha C_{A} \zeta \frac{Y}{X} \mu_{Y}+\left(1+\alpha C_{G}\right) x_{G}\left(\mu_{G}-r\right)+\alpha \frac{1}{X} C_{Z}^{\prime} I_{Z}\left(\mu_{Z}-r 1_{K}\right)\right] \mathrm{d} t } \\
& +\left[\alpha C_{A} x_{S}^{\prime} \sigma_{S}+\alpha C_{A} \zeta \frac{Y}{X} \sigma_{Y}+\left(1+\alpha C_{G}\right) x_{G} \sigma_{G}+\alpha \frac{1}{X} C_{Z}^{\prime} I_{Z} \sigma_{Z}\right] \mathrm{d} B
\end{aligned}
$$

The system of first order conditions for an optimum follows: 


$$
\begin{aligned}
0_{N}= & {\left[\left(\alpha C_{A}\right)\left(\mu_{S}-r 1_{N}\right)\right] J_{X} X } \\
& +\left[\left(\alpha C_{A}\right)^{2} \Sigma_{S S} x_{S}+\left(\left(\alpha C_{A}\right)^{2} \zeta \frac{Y}{X}\right) \Sigma_{S Y}+\left(\alpha C_{A}\left(1+\alpha C_{G}\right) x_{G}\right) \Sigma_{S G}\right. \\
& \left.+\left(\alpha^{2} C_{A} \frac{1}{X}\right) \Sigma_{S Z} I_{Z}^{\prime} C_{Z}\right] J_{X X} X^{2}+\left[\left(\alpha C_{A}\right) \Sigma_{S Z}\right] I_{Z}^{\prime} J_{X Z} X
\end{aligned}
$$

The optimal vector of proportions $x_{S}$ then writes:

$$
\begin{aligned}
x_{S}= & -\left(\Sigma_{S S}\right)^{-1}\left(\mu_{S}-r 1_{N}\right) \frac{J_{X}}{J_{X X} X} \frac{1}{\alpha C_{A}}-\left(\Sigma_{S S}\right)^{-1} \Sigma_{S Y} \zeta \frac{Y}{X} \\
& -\left(\Sigma_{S S}\right)^{-1} \Sigma_{S G} x_{G} \frac{1+\alpha C_{G}}{\alpha C_{A}}-\left(\Sigma_{S S}\right)^{-1} \Sigma_{S Z} I_{Z}^{\prime} C_{Z} \frac{1}{C_{A}} \frac{1}{X} \\
& -\left(\Sigma_{S S}\right)^{-1} \Sigma_{S Z} I_{Z}^{\prime} J_{X Z} \frac{1}{J_{X X} X} \frac{1}{\alpha C_{A}}
\end{aligned}
$$

\subsubsection{Result interpretation}

Five components form the optimal investment strategy: the modified speculative fund, the preference independent wage-hedge term, the modified preference independent guarantee-hedge fund and two modified state variable-hedge terms, from which one depends on the investor's preferences and the other does not.

Let us begin with the modified speculative component, and first assume that $\alpha=1$, which allows to compare the general cases of internal and external guarantees. When going from the first to the second one, $C_{A}$ appears in the denominator of the speculative component. The delta of a call taking a value between 0 and 1 , the absolute value of the speculative term increases. The granting of an external guarantee thus leads to a riskier behavior of the investor.

We now turn to the analysis of the case of $0<\alpha<1$. One observes that the riskiness of the investor's behavior increases when $\alpha$ decreases, $\alpha$ being defined as the proportion of the fund surplus belonging to the participant. Adopting a surplus sharing rule will thus have crucial implications for the optimal asset allocation of the investor. We conclude that, when the participant does not include the solvency constraint in his optimization program, such a rule should not be introduced, because it proves to be dangerous for the institution guaranteeing the payment of the possible deficit.

The preference independent wage-hedge fund is the only portfolio component to remain unchanged when turning form the case of an internal guarantee to the case of an external one. Neither the presence of the call in the scheme final payoff, nor the surplus sharing rule do not influence its value.

As to the modified preference independent guarantee-hedge fund, let us first note that its sign changes, when going from the internal guarantee case to the external guarantee one: The impact of an external guarantee on the participant's behavior, as to the guarantee-hedge term, proves to be opposite to the one characteristic of an internal guarantee. Second, one observes that the term is now multiplied by $\frac{1+\alpha C_{G}}{\alpha C_{A}}$ : 
The transition to an external guarantee once again leads to the presence of $C_{A}$ in the denominator and the guarantee-hedge term also increases (in absolute value) when $\alpha$ decreases.

Let us now decompose this modified guarantee-hedge term in two sub-elements: $-\left(\Sigma_{S S}\right)^{-1} \Sigma_{S G} x_{G} \frac{1}{\alpha C_{A}}-\left(\Sigma_{S S}\right)^{-1} \Sigma_{S G} x_{G} \frac{C_{G}}{C_{A}}$. The first sub-element then refers to the variable $G$ defining the first term of the variable $X$ definition. It reproduces the structure of the guarantee-hedge fund characteristic of the internal guarantee case, except that it is multiplied by the term $\frac{1}{\alpha C_{A}}$. The second sub-element results from the presence of the variable $G$ in the definition of the call incorporated in the final payoff of the scheme. It also reproduces the structure of the guarantee-hedge term characteristic of the internal guarantee case, except that it is multiplied by $C_{G}$ and divided by $C_{A}$. $\alpha$ vanishes, which means that this second sub-element is independent from the surplus sharing rule. To conclude, $-\left(\Sigma_{S S}\right)^{-1} \Sigma_{S G} x_{G} \frac{C_{G}}{C_{A}}$ is a hedge portfolio against variations in a variable determining for the value of the call. The presence of an option in the objective function thus leads to the presence of a specific term in the optimal investment strategy, which is absent from the no-option case.

The two last terms in the optimal investment strategy define modified hedging components against state variable variations. The first component, preference independent, exists because state variables influence the value of the call; The second one, preference dependent, is present because the indirect utility function has state variables as its argument. The call-related component, inexistent in the nooption (or internal guarantee) case, has a structure similar to the one of the second sub-element of the guarantee-hedge term. The preference-dependent component reproduces the structure of the corresponding element in the internal guarantee case, except that it is multiplied by $\frac{1}{\alpha C_{A}}$.

\section{Conclusion}

This article defines the optimal asset allocation of the main types of pension funds. It proposes a new framework for comparing the strategies of the different funds, which is based on the nature of the minimum guarantee offered by or to pension funds. If the guarantee is internal (i.e. the fund has to generate a minimum return), the fund manager must conduct an investment policy guaranteeing that the final asset value lies above the final guarantee value: The utility function argument then takes the form of the surplus of the fund assets over the guarantee. The resulting optimal policy resembles the one adopted by a portfolio insurer. In the case of a guarantee offered by an external institution, the investor receives the maximum of the final asset value and the final guarantee value. The solvency constraint is absent from the optimization program. As a consequence, an option appears in the utility function argument.

The topic of external guarantees with regard to their consequences for the optimal portfolio policy has not attracted attention in the literature yet. One of the reasons seems to lie in the pension fund-specific character of this sort of guarantees. As pension funds manage a wealth whose purpose is to maintain an adequate 
standard of living during the retirement period, it seems natural for the employer or the State to become involved in order to guarantee that this standard is sufficiently high.

The optimization programs of DB, DC and TMP schemes are analyzed. The program definition shows that the type of the plan constitutes only one of the determining factors. It also depends on the person bearing the responsibility for the possible deficit, the presence and the nature of guarantees and the introduction of a surplus sharing rule. By comparing the programs, one notices some interesting similarities between the different types of schemes. First, it turns out that the optimal policy structure of a constrained DB plan (i.e. subject to the solvency constraint), of a DC plan subject to an internal guarantee and of a TMP plan is similar, because the three plans follow a portfolio insurance strategy. The difference concerns the nature of the variable the variations of which one must hedge against. Furthermore, if a TMP plan targets the value of the DB pension, the constrained DB plan and the TMP plan conduct an identical allocation policy.

The analysis made in this article shows that it is not necessary to study the portfolio choice behavior of each type of fund separately. By choosing an adapted framework, one has the possibility to analyze and compare the policies of the different types of funds in a unique setting. The one chosen here, based on the differentiation between the guarantee nature, seems well suited for such a purpose.

The internal guarantee optimal allocation has the four following components: the speculative term, two preference independent terms-the salary-hedge portfolio (resulting from the definition of the contributions) and the guarantee-hedge oneand a preference dependent state variable-hedge component. An interesting result is that the case of a DB scheme leads to an increased role of the wage in defining the optimal policy, because this variable determines the second as well as the third term of the optimal strategy.

The derivation of the external guarantee optimal policy, which uses the principles of standard options theory, constitutes a contribution of this article. When compared with the internal guarantee strategy, all the components, except the salary-hedge term, are divided by the delta of the call incorporated in the final wealth definition. The speculative term absolute value increases: The investor's behavior becomes riskier. Furthermore, the guarantee-hedge term changes its sign. Finally, the presence of the call in the objective function leads to the presence of two additional hedge terms in the investment strategy, covering against guarantee and state variable variations.

One of the main results of this article states that the optimal strategy becomes riskier when the guarantee is external. This confirms the view of Tamburi and Chassard [2002]: "Too many compensatory guarantees do not encourage a prudential behavior of actors." We emphasize that the analysis of the behavior induced by this type of contracts should precede the introduction of such guarantees.

Acknowledgements I am very grateful to Patrice Poncet for extensive discussions on an earlier version of this article. I would like to thank Radu Vranceanu for very valuable suggestions. I have also 
benefited from helpful comments from Damien Besancenot, Jean-François Boulier, Hubert de la Bruslerie, Najat El Mekkaoui de Freitas, Steven Haberman, Constantin Mellios, Roland Portait, François Quittard-Pinon and the participants of the 4th Meeting on Social Security and Complementary Pensions Systems in Lisbon in May 2005, the International Conference of the French Finance Association in Paris in June 2005 and the Annual Meeting of the Financial Management Association in Chicago in October 2005. I wish to thank two anonymous referees, whose analyses permitted to substantially improve the quality of the article.

\section{References}

BASAK, S. [2002]: “A Comparative Study of Portfolio Insurance,” Journal of Economic Dynamics and Control, 26, 1217-1241.

BATTOCCHIO, P. and MENONCIN, F. [2004]: "Optimal Pension Management in a Stochastic Framework," Insurance: Mathematics and Economics, 34, 79-95.

BATTOCCHIO, P., MENONCIN, F., and SCAILlET, O. [2007]: "Optimal Asset allocation for Pension Funds Under Mortality Risk During the Accumulation and Decumulation Phases," Annals of Operations Research, 152(1), 141-165.

BLACK, F. and SCHOLES, M. [1973]: “The Pricing of Options and Corporate Liabilities," Journal of Political Economics, 81, 637-654.

BLAKE, D. [1998]: "Pension Schemes as Options on Pension Fund Assets: Implications for Pension Fund Management," Insurance: Mathematics and Economics, 23, 263-286.

BOULIER, J.-F., HUANG, S., and TAILLARD, G. [2001]. Optimal Investment Under Stochastic Interest Rates: The Case of a Protected Defined Contribution Pension Fund," Insurance: Mathematics and Economics, 28, 173-189.

CAIRNS, A., BLAKE, D., and DOWD, K. [2006]: "Stochastic Lifestyling: Optimal Dynamic Asset allocation for Defined Contribution Pension Plans," Journal of Economic Dynamics and Control, 30(5), 843-877.

COX, J.C. and HUANG, C. [1989]: "Optimal Consumption and Portfolio Policies When Asset Prices Follow a Diffusion Process,” Journal of Economic Theory, 49, 33-83.

COX, J.C. and HUANG, C. [1991]: "A Variational Problem Arising in Financial Economics," Journal of Mathematical Economics, 20, 465-487.

DEELSTRA, G., GRASSELLI, M., and KOEHL, P.-F. [2003]: "Optimal Investment Strategies in the Presence of a Minimum Guarantee," Insurance: Mathematics and Economics, 33, 189-207.

KARATZAS, I., LEHOCZKY, J., SETHI, S., and SHREVE, S. [1986]: "Explicit Solution of a General Consumption/Investment Problem," Mathematics of Operations Research, 11(2), 261-294.

LIOUI, A. and PONCET, P. [2003]: "Dynamic Asset Pricing with Non-redundant Forwards,” Journal of Economic Dynamics and Control, 27, 1163-1180.

MARGRABE, W. [1978]: "The Value of an Option to Exchange One Asset for Another," Journal of Finance, 33(1), 177-186.

MENONCIN, F. [2002]: "Optimal Portfolio and Background Risk: An Exact and An Approximated Solution," Insurance: Mathematics and Economics, 31, 249-265.

MENONCIN, F. and SCAILLET, O. [2003]: "Mortality Risk and Real Optimal Asset Allocation for Pension Funds," FAME Research Paper No. 101, September.

MERTON, R.C. [1971]: "Optimum Consumption and Portfolio Rules in a Continuous-Time Model," Journal of Economic Theory, 3, 373-413.

MERTON, R.C. [1990]: Continuous-Time Finance, Cambridge: Blackwell.

RUDOLF, M. and ZIEMBA, W.T. [2004]: “Intertemporal Surplus Management," Journal of Economic Dynamics and Control, 28, 975-990.

SUNDARESAN, S. and ZAPATERO, F. [1997]: "Valuation, Optimal Asset Allocation and Retirement Incentives of Pension Plans," Review of Financial Studies, 10(3), 631-660.

TAMBURI, G. and CHASSARD, Y. [2002]: Fonds De Pension, Comment font les autres pays. Economica, Paris. 